\title{
ART-2 ARTIFICIAL NEURAL NETWORKS APPLICATIONS FOR CLASSIFICATION OF VIBRATION SIGNALS AND OPERA-TIONAL STATES OF WIND TURBINES FOR INTELLIGENT MONITORING
}

\author{
Tomasz BARSZCZ ${ }^{1}$, Andrzej BIELECKI², Mateusz WÓJCIK ${ }^{3}$, Marzena BIELECKA $^{4}$ \\ ${ }^{1}$ AGH University of Science and Technology, Faculty of Mechanical Engineering and Robotics, \\ al.Mickiewicza 30, 30-059 Cracow, Poland, tbarszcz@agh.edu.pl \\ ${ }^{2}$ AGH University of Science and Technology, Faculty of Electrotechnics, Automation, Computer Science and \\ Biomedical Engineering, al. Mickiewicza 30, 30-059 Cracow, Poland azbielecki@gmail.com \\ ${ }^{3}$ Jagiellonian University, Faculty of Physics, Astronomy and Applied Computer Science, \\ ul. Reymonta 4, 30-059 Cracow, Poland, mateusz.wojcik@uj.edu.pl \\ ${ }^{4}$ AGH University of Science and Technology, Faculty of Geology, Geophysics and Environmental \\ Protectional.Mickiewicza 30, 30-059 Cracow, Poland, bielecka@agh.edu.pl
}

\begin{abstract}
Summary
In recent years wind energy is the fastest growing branch of the power generation industry. The largest cost for the wind turbine is its maintenance. A common technique to decrease this cost is a remote monitoring based on vibration analysis. Growing number of monitored turbines requires an automated way of support for diagnostic experts. As full fault detection and identification is still a very challenging task, it is necessary to prepare an "early warning" tool, which would focus the attention on cases which are potentially dangerous. There were several attempts to develop such tools, in most cases based on various classification methods. As the ART neural networks are capable to perform efficient classification and to recognize new states when necessary, they seems to be a proper tool for classification of vibration signals of bearing in gears in wind turbines. The verification of ART-2 networks efficiency in this task is the topic of this paper.
\end{abstract}

Keywords: wind turbines, monitoring, ART neural network

\section{INTRODUCTION}

In recent years wind energy is the fastest growing branch of the power generation industry. The average yearly growth in the years 1997-2003 achieved $32 \%$ in the United States and 22\% in the European Union [4] and these figures will hold for at least the next decade. The distribution of costs during the life cycle of the unit for wind energy is significantly different from that of traditional, fossil fired units [4]. First of all, initial investment costs are relatively higher, whereas in traditional units cost of fuel plays important role (usually it is the second largest cost). After commissioning, the largest cost for the wind turbine (WT for abbreviation) is maintenance. With proper maintenance policies, wind turbines can achieve the highest level of availability in the power generation sector - even up to $98 \%$.

Therefore, condition monitoring of wind turbines, including fault diagnostics, in particular at the early stage of a fault occurrence or even participatory actions, is an essential problem in wind turbines engineering in particular $[7,9,12]$ and in rotating machinery engineering in general [1]. There were several attempts to develop various monitoring tools, in most cases based on various classification methods. Some of them are based on artificial neural networks (ANNs for abbreviation). In most types of ANNs the learning process is unsuitable for cases of continuous machinery intelligence monitoring. This means, among others, that adding new patterns as inputs requires repetition of the learning process. In ART networks, introduced by Car- penter and Grossberg $[5,6]$, the learning process is not separated from its operation. Furthermore, ART neural networks are capable to add new states when necessary $[11,13,16]$. Therefore, this sort of ANNs was tested as a tool for classification of states in continuous monitoring.

\section{THE MACHINE DESCRIPTION}

The faults which are sought in wind turbines are primarily of mechanical origin. The wind turbine with the gearbox, which is the most popular type, can be described in the following way. The main rotor with three blades is supported by the main bearing and transmits the torque to the planetary gear. The main rotor is connected to the plate which is the gear input. The planetary gear has three planets, with their shafts attached to the plate. The planets roll over the stationary ring and transmit the torque to the sun. The sun shaft is the output of the planetary gear. The sun drives the two-stage parallel gear which has three shafts: the slow shaft connected to the sun shaft, the intermediate shaft and the fast shaft, which drives the generator. The overall gear ratio is in the range of 1:100. The generator produces alternating current of slightly varying frequency. This current is converted first into direct current power and then into alternating current power of frequency equal to the grid frequency. Electric transformations are performed by the controller at the base of the tower - see Fig. 1. 


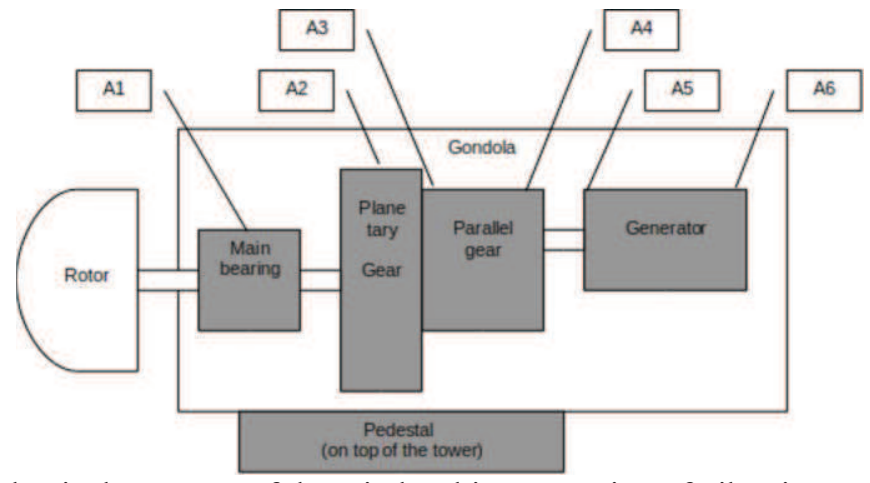

Fig. 1. The mechanical structure of the wind turbine. Location of vibration measurement sensors is shown by An symbols

In the field of vibrodiagnostics, a machine operational state is understood as an accepted range of machines operational points enabling referential analysis. In practice, machine operating point is defined by values of available measurements of physical quantities such as speed, load, pressure, temperature, etc., usually called machine process parameters [8]. Typically, from each vibration record, a set of diagnostic indicators is calculated known as trends. Each trend point is a combination of representation of true machine technical condition and behaviour, machines current operating point, measurement error and random factor. In a typical condition monitoring set up, each trend is tracked against a precalculated threshold value. In this case, operational states (shortly called states) are used for data classification during the data acquisition process. On the basis of these states, data is combined into sets, which are assumed to represent a particular machine. Consequently, the overall number of defined diagnostic indicators and estimators is equal to the number of indicators and estimators multiplied by the number of states. Therefore, from operator point of view, it is desirable to have as little states as possible. On the other hand, from reliable-diagnostics point of view, in order to minimize the fluctuation of machines operating points, it is desirable to define ranges of states as low as possible. In this case, the state configuration would result either in (i) single operational state with low permissible fluctuation of operational parameters, (ii) a large number of operational states with low permissible fluctuations of operational parameters.

\section{CLASSIFICATION PROBLEM OF WIND TURBINE VIBRATION SIGNALS AND OPERATIONAL STATES}

In recent years large development of monitoring and diagnostic technologies for WTs has taken place. The growing number of installed systems created the need for analysis of gigabytes of data created every day by these systems. Apart from the development of several advanced diagnostic methods for this type of machinery there is a need for a group of methods, which will act as an "early warning". The idea of this approach could be based on a data driven algorithm, which would decide on a similarity of current data to the data, which are already known. In other words, the data from the turbine should be accounted for one of known states. If this is a state describing a failure, the human expert should be alarmed. If this is an unknown state, the expert should be informed about the situation and asked for a definition of such a new state.

This approach could be called "the blunt expert", which maybe sounds strange, but gives the most important feature of the proposed method. This approach may break the biggest barrier of application of artificial neural networks (ANNs) in diagnostics, which is availability of significant amount of training data. As in real cases it is not possible to acquire it, it is only possible to train ANNs for a few cases covered by available data.

The problem of classification was investigated by several authors. One of the first works was research by Shuhui et al. [15], who compared classification techniques for the wind curve estimation. This work was often referenced by others, but from the ANN point of view it only multilayered feed-forward types of networks. Another important contribution was given by Kim [10], who compared performance of several classification methods. His experiments showed that unless the number of independent variables in the system is low, ANNs perform better than other methods. Again, the investigated network was the multi-layer feedforward network trained by the back-propagation algorithm.

There are no works, known to us, which would consider application of ART networks for the classification of WT states. There were also works applying wind turbines for wind and power generation prediction, but this issue is outside the scope of this paper.

As the ART networks are capable to perform efficient classification and to recognize new states when necessary $[5,6]$, we performed research of initial classification task. The goal of the experiment was verification of ART classification capabilities with comparison to the human expert. This type of data is acquired in the majority of cases and the successful classifier should create a reasonable number of classes, similar to these by a human expert. This task is the main goal of the following paper.

As such a classification was shown, it is thus possible to filter out states, which are known to be correct. 
The expert can then focus only on "suspicious" states returned by the algorithm.

\section{CHARACTERISTICS OF THE ART-2 NEURAL NETWORKS}

Let us briefly recall ART-2 neural network properties $[3,6,14]$. The ART-2 is an unsupervised neural network based on adaptive resonance theory (ART). A typical ART-2 architecture, introduced by Carpenter and Grossberg [5, 6], is presented in Fig.2 (only one unit of each type is shown here). In the attentional subsystem, an input pattern $s$ is first presented to the $F_{1}$ layer, which consists of six kinds of units - the $W, X, U$, $V, P$ and $Q$ cells. It then undergoes a process of activation, including normalization, noise suppression and updating. This results in an output pattern $p$ from the $F_{I}$ layer. Responding to this output pattern, an activation is produced across $F_{2}$ layer through bottom-up weights $b_{i j}$. As the $F_{2}$ layer is a competitive layer with a winner-takes-all mode, only one stored pattern is a winner. It also represents the best matching pattern for the input pattern at the $F_{l}$ layer. Furthermore, the pattern of activation on the $F_{2}$ layer brings about an output pattern that is sent back to the $F_{l}$ layer via top-down weights $t_{j i}$. For the orienting sub-system, it contains a reset mechanism $\mathrm{R}$ and a vigilance parameter $q$ to check for the similarity between the output pattern from the $F_{2}$ layer and the original input pattern from the $F_{l}$ layer. If both patterns are concordant, the neural network enters a resonant state where the adaptation of the stored pattern is conducted. Otherwise, the neural network will assign an uncommitted (inhibitory) node on the $F_{2}$ layer for this input pattern, and thereafter, learn and transform it into a new stored pattern.

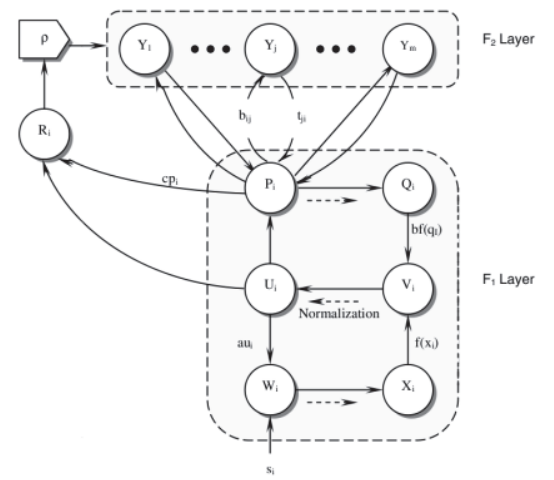

Fig. 2 ART-2 architecture

\section{RESULTS}

A simulation of a wind turbine work using historical data has been done. Changes of operational states and vibration signals were investigated. Historical data contain 27000 measuring points in time (sampling frequency is 1 per 15 minutes). Each point has three operational states values and one vibration signal value. Simulation step was taken every 1000 measuring points. ART-2 network (described in section 4) was used in the simulation in a following way.

1. ART-2 network is initialized.

2. Time $t$ as a pointer of measuring point is set to 1000 .

3. All measuring points' values are scaled to $[0,1]$.

4. While $t$ is not greater then 27000

a. ART-2 learning process is performed for each measuring point in $[1, \mathrm{t}]$. The order of points is randomized. Each point is taken once. The vigilance parameter is set to 0.982 .

b. Figures of classification states are plotted. Each network pattern determines some state of machine.

Figure 3 presents how the ART-2 network has classified states for first 5000 measuring points. There were 3 dominate states. After 15000 measuring points 4-th dominate state has joined. That situation is presented on figure 4 . At the end classification states look like in the figure 5 .

Table 1 describes how numbers of classified states were changing during simulation process.

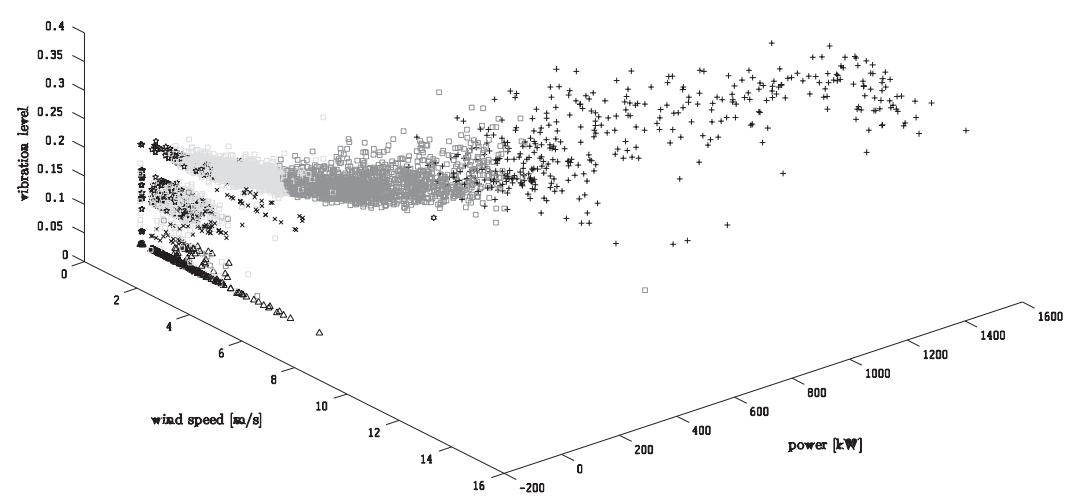

Fig. 3 The operational states (wind, power) and vibration signal classified using ART-2 network using 5000 measuring points. 


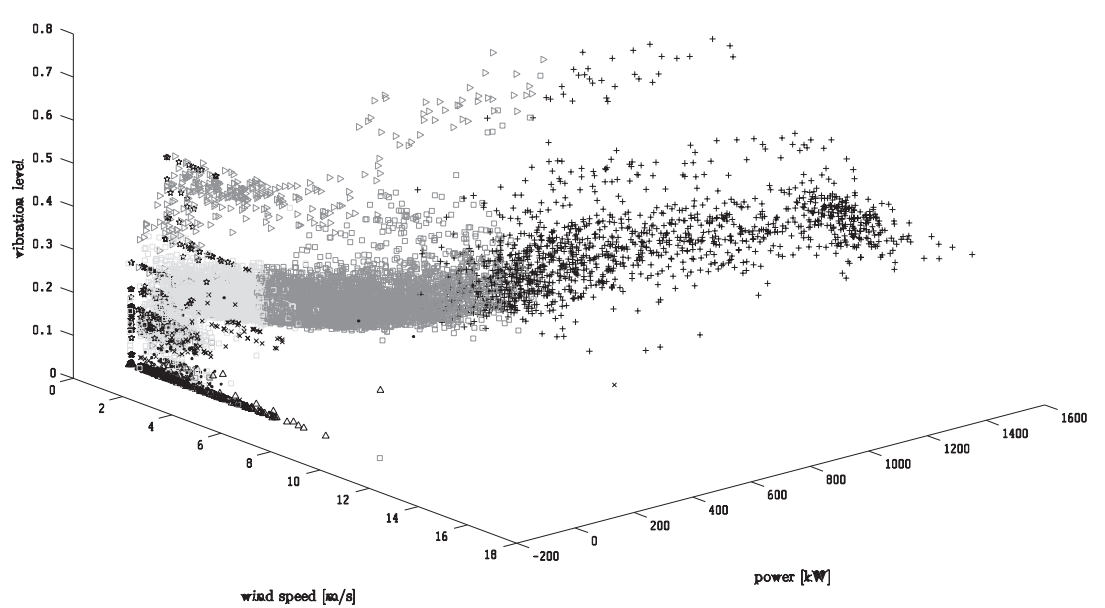

Fig. 4 The operational states (wind, power) and vibration signal classified using ART-2 network using 15000 measuring points.

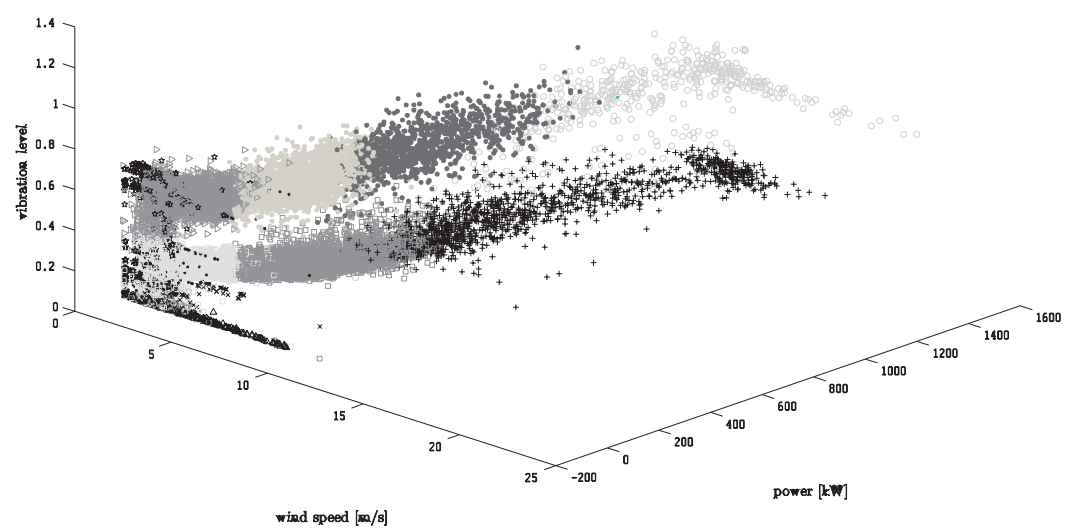

Fig. 5 The operational states (wind, power) and vibration signal classified using ART-2 network using 27000 measuring points.

Table 1 Details of simulation steps. Column 1 (Details) - number of processed measuring points. Column 2 - number of detected states. Values in column 3-17 represent numbers of measuring point which were classified as a specified state. Last column - number of states with value greater than 10.

\begin{tabular}{|c|c|c|c|c|c|c|c|c|c|c|c|c|c|c|c|c|c|}
\hline Distance & States & s \#1 & $\# 2$ & $\# 3$ & $\# 4$ & $\# 5$ & $\# 6$ & $\# 7$ & $\# 8$ & $\# 9$ & $\# 10$ & $\# 11$ & $\# 12$ & $\# 13$ & $\# 14$ & $\# 15$ & $\#>10$ \\
\hline 1000 & 5 & 677 & 91 & 185 & 18 & 29 & & & & & & & & & & & 5 \\
\hline 2000 & 6 & 1354 & 131 & 380 & 33 & 98 & 4 & & & & & & & & & & 5 \\
\hline 3000 & 6 & 2033 & 144 & 602 & 70 & 147 & 4 & & & & & & & & & & 5 \\
\hline 4000 & 7 & 2560 & 184 & 751 & 119 & 366 & 9 & 11 & & & & & & & & & 6 \\
\hline 5000 & 7 & 2170 & 210 & 1656 & 208 & 447 & 10 & 299 & & & & & & & & & 6 \\
\hline 6000 & 8 & 2269 & 238 & 2348 & 217 & 505 & 7 & 412 & 4 & & & & & & & & 6 \\
\hline 7000 & 8 & 2590 & 257 & 2776 & 234 & 595 & 7 & 535 & 6 & & & & & & & & 6 \\
\hline 8000 & 8 & 3106 & 252 & 3018 & 233 & 620 & 9 & 755 & 7 & & & & & & & & 6 \\
\hline 9000 & 8 & 3531 & 281 & 3250 & 264 & 725 & 12 & 922 & 15 & & & & & & & & 8 \\
\hline 10000 & 9 & 4008 & 256 & 3702 & 256 & 666 & 113 & 977 & 20 & 2 & & & & & & & 8 \\
\hline 11000 & 10 & 4362 & 240 & 4099 & 248 & 486 & 473 & 1053 & 24 & 14 & 1 & & & & & & 9 \\
\hline 12000 & 11 & 4594 & 247 & 4551 & 292 & 537 & 593 & 1137 & 32 & 14 & 1 & 2 & & & & & 9 \\
\hline 13000 & 11 & 4938 & 256 & 4964 & 292 & 619 & 624 & 12 & 39 & 14 & 2 & 3 & & & & & 9 \\
\hline 14000 & 11 & 5116 & 273 & 5369 & 293 & 801 & 776 & 1303 & 44 & 14 & 4 & 7 & & & & & 9 \\
\hline 15000 & 11 & 5418 & 277 & 5283 & 314 & 1129 & 858 & 1345 & 59 & 14 & 7 & 296 & & & & & 10 \\
\hline 16000 & 11 & 5425 & 280 & 5296 & 384 & 1211 & 972 & 1358 & 129 & 14 & 10 & 921 & & & & & 10 \\
\hline 17000 & 12 & 5515 & 260 & 5276 & 339 & 12171 & 1336 & 1317 & 269 & 12 & 23 & 1433 & 3 & & & & 11 \\
\hline 18000 & 12 & 5574 & 261 & 5287 & 344 & 12371 & 1346 & 1350 & 274 & 12 & 28 & 2283 & 4 & & & & 11 \\
\hline 19000 & 12 & 5635 & 279 & 5244 & 354 & 13281 & 1475 & 1353 & 273 & 12 & 35 & 3006 & 6 & & & & 11 \\
\hline 20000 & 12 & 5633 & 292 & 5268 & 386 & 14401 & 1573 & 1350 & 291 & 12 & 46 & 3700 & 9 & & & & 11 \\
\hline 21000 & 13 & 5520 & 298 & 5282 & 399 & 14431 & 16 & 13 & 288 & 13 & 91 & 3895 & 801 & 11 & & & 13 \\
\hline 22000 & 14 & 5299 & 29 & 541 & 408 & 14 & 15 & 13 & 291 & 12 & 174 & 29772 & 2306 & 497 & 4 & & 13 \\
\hline 23000 & 14 & 5151 & 302 & 5515 & 484 & 14 & 16 & 13 & 315 & 12 & 182 & 3416 & 2546 & 545 & 5 & & 13 \\
\hline 24000 & 14 & 5228 & 314 & 54 & 481 & 14991 & 16 & 13 & 331 & 12 & 200 & 37812 & 2979 & 653 & 5 & & 13 \\
\hline 25000 & 14 & 5233 & 323 & 5499 & 511 & 15471 & 1717 & 1350 & 337 & 12 & 217 & 4283 & 3262 & 700 & 9 & & 13 \\
\hline 25000 & 14 & 5226 & 343 & 5512 & 578 & 16381 & 1782 & 1349 & 340 & 12 & 223 & 4718 & 3554 & 710 & 15 & & 14 \\
\hline 27000 & 15 & 5177 & 346 & 5524 & 572 & 16361 & 1791 & 1394 & 341 & 13 & 229 & 52313 & 3737 & 882 & 30 & 97 & 15 \\
\hline
\end{tabular}




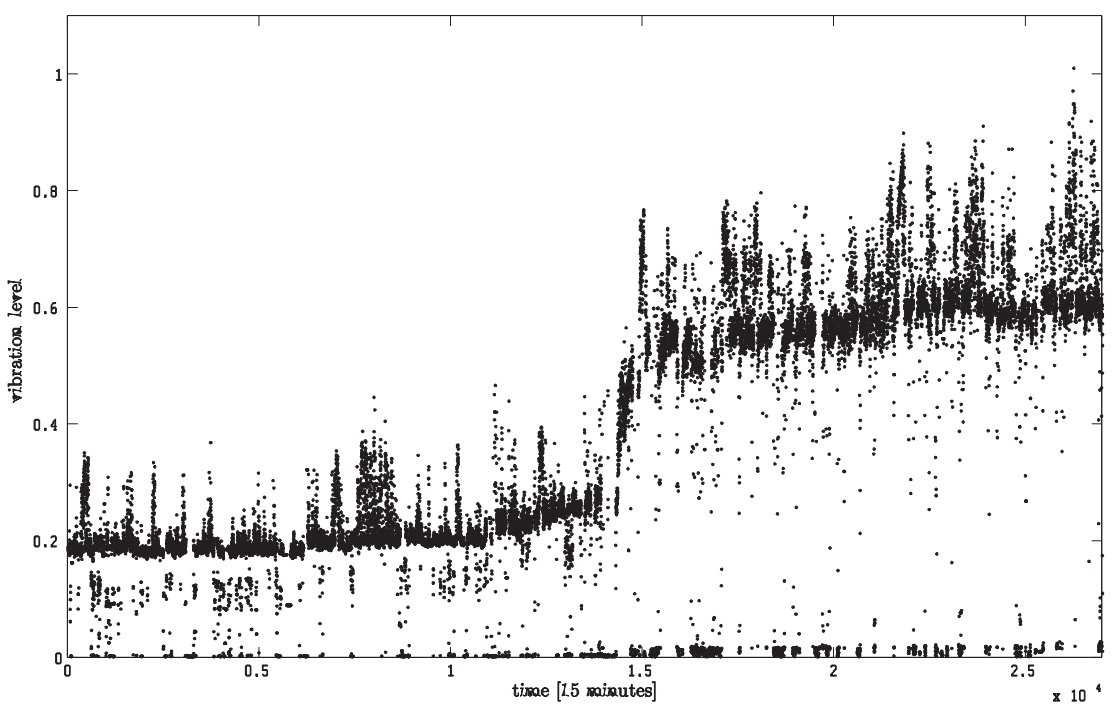

Fig. 4 Vibration signals for all measuring points.

Looking at last the column there can be observed first changes of numbers of states. First change has happened after 8000 measuring points. Next ones after 10000 and after 14000 points. Figure 6 presents the vibration signals for all measuring points (ordered by time). After about 14000 points the biggest damage happened. There must have been some symptoms of that damage. Mentioned two first changes could be candidates of those symptoms.

\section{CONCLUDING REMARKS}

As it has been mentioned, monitoring is crucial in wind turbines exploitation. On the other hand, there are very few attempts to create system for intelligent monitoring based on artificial intelligence - see [7] and references given there. The experiments described in this paper show that ART ANNs can be effective tool for such task performing - the symptoms of a turbine damage can be detected using ART ANN. It should be stressed however, that the obtained results are preliminary ones - only one vibration channel has been used whereas, usually, a few vibration channels are observed simultaneously - see [2]. The monitoring module based on ANNs is planned to be a module of expert system for intelligent monitoring and fault diagnostics in wind turbines.

\section{ACKNOWLEDGEMENTS}

The paper was supported by the Polish Ministry of Science and Higher Education under Grant No. N504 147838 .

\section{REFERENCES}

1. Barszcz T., Bielecki A., Romaniuk T. (2009) Application of probabilistic neural networks for detection of mechanical faults in electric motors. Electrical Review 8/2009, 37-41.

2. Barszcz T., Bielecka M., Bielecki A., Wójcik M. (2012) Wind speed modeling using Weierstrass function fitted by a genetic algorithm. Journal of
Wind Engineering and Industrial Aerodynamics 109, 68-78.

3. Barszcz T., Bielecki A., Wójcik M. (2012) ARTtype artificial neural networks applications for classification of operational states in wind turbines. Lecture Notes in Artificial Intelligence 6114, 11-18

4. Barszcz T., Randall R. B. (2009) Application of spectral kurtosis for detection of a tooth crack in the planetary gear of a wind turbine. Mechanical Systems and Signal Processing 23, 1352-1365.

5. Carpenter G. A., Grossberg S. (1987) A massively parallel architecture for a self-organizing neural pattern recognition machine. Computer Vision, Graphics, and Image Processing 37, 54-115.

6. Carpenter G. A., Grossberg S. (1987) ART2: selforganization of stable category recognition codes for analog input pattern. Applied Optics 26, 49194930.

7. Hameeda Z., Honga Y. S., Choa T. M., Ahnb S. H., Son C. K. (2009) Condition monitoring and fault detection of wind turbines and related algorithms: $A$ review, Renewable and Sustainable Energy Reviews, 13, 1-39.

8. Jabłoński A., Barszcz T. (2012) Procedure for data acquisition for machinery working under nonstationary operational conditions, The Ninth International Conference on Condition Monitoring and Machinery Failure Prevention Technologies, 12-14 June 2012, London.

9. Jabłoński A., Barszcz T., Bielecka M. (2011) Automatic validation of vibration signals in wind farm distributed monitoring systems, Measurement, vol.44, 1954-1967.

10. Kim Y. S., Performance evaluation for classification methods: A comparative simulation study.

11. Korbicz J., Obuchowicz A., Uciński D. (1994) Artificial Neural Networks. Foundations and Applications. Academic Press PLJ, Warsaw (in Polish). 
12. Kusiak A., Li W. (2011) The prediction and diagnosis of wind turbine faults, Renewable Energy, vol.36, 2011, 16-23.

13. Rutkowski L. (1996) Neural Networks and Neurocomputers. Technical University in Cze,stochowa Press, Częstochowa (in Polish).

14. Shieh M. D., Yan W., Chen C. H. (2008) Soliciting customer requirements for product redesign based on picture sorts and ART2 neural network. Expert Systems with Applications 34, 194-204.

15. Shuhui L., Wunsch D. C., O'Hair E., Giesselmann M.G. (2001) Comparative analysis of regression and artificial neural network models for wind turbine power curve estimation, Journal of Solar Energy Engineering 123, 327-332.

16. Tadeusiewicz R. (1993) Neural Networks. Academic Press, Warsaw (in Polish). 